\title{
Nuevo dispositivo de micronebulización con control continuo de la fracción inspirada de oxígeno (“CONTROLizer”)
}

\author{
New micronebulizer device with continuous control \\ of the inspired oxygen fraction ("CONTROLizer")
}

\author{
Mauricio Orozco-Levi, Alba Ramírez-Sarmiento, Angela Espitia, \\ Maria Fernanda Saavedra, Melissa Mogollón, Diana Isabel Cáceres, \\ Rubén Duarte, Norma Serrano, Leonardo Rodriguez, \\ Eugenio Sarmiento, Camilo Pizarro - Floridablanca (Colombia) \\ JoAquim Gea • Barcelona (EsPaña)
}

\section{Resumen}

Una de las técnicas más comunes de tratamiento respiratorio es la nebulización de medicamentos para administrarlos por vía inhalada utilizando oxígeno como propelente, el cual a su vez es considerado un fármaco que puede generar efectos adversos relacionados con la dosificación. El objetivo de esta investigación fue diseñar, desarrollar y analizar un sistema dual de micronebulización con control preciso de la $\mathrm{FIO}_{2}$.

Métodos: se diseñó, construyó y evaluó un modelo virtual y un prototipo funcional siguiendo la metodología de bioingeniería de un nuevo dispositivo de micronebulización con control de $\mathrm{FIO}_{2}$. Se evaluó el funcionamiento del dispositivo y su impacto en voluntarios sanos y pacientes hospitalizados mediante escalas psicométricas específicas.

Resultados: se conceptualizó, diseñó y construyó un dispositivo que integra un micronebulizador (recipiente), que permite nebulizar suero fisiológico o soluciones de diversos fármacos basado en la presurización de un gas propelente (aire u oxígeno) junto a un mecanismo de ajuste de la fracción inspirada de $\mathrm{O}_{2}$ (mínimo 21\%; máximo 99\%). Los límites (máximo y mínimo) de flujo recomendado para generar el aerosol son 6-8 1/min. En ningún caso de uso se presentaron complicaciones.

Conclusiones: CONTROLizer es un dispositivo integrado dual y no-invasivo para la micronebulización de soluciones respiratorias y control continuo de la fracción inspirada de oxígeno. Por sus cualidades y funcionamiento, el dispositivo fue percibido como adecuado y seguro para aplicarse en individuos sanos y pacientes ingresados tanto en salas de cuidados intensivos como de hospitalización convencional. (Acta Med Colomb 2018; 43: 90-99).

Palabras clave: oxigenoterapia, insuficiencia respiratoria, hipercapnia, dispositivos médicos, micronebulización.

\footnotetext{
Abstract

One of the most common techniques of respiratory treatment is the nebulization of medications to be administered by inhalation using oxygen as a propellant, which in turn is considered a drug that can generate adverse effects related to the dosage. The objective of this research was to design, develop and analyze a dual micronebulization system with precise control of $\mathrm{FIO}_{2}$.

Methods: a virtual model and a functional prototype were designed, constructed and evaluated following the bioengineering methodology of a new micronebulizer device with $\mathrm{FIO}_{2}$ control. The functioning of the device and its impact on healthy volunteers and hospitalized patients were evaluated through specific psychometric scales.

Results: a device that integrates a micronebulizer (container), which allows to nebulize physiological saline or solutions of diverse drugs based on the pressurization of a propellant gas (air or oxygen) along with a mechanism of adjustment of the fraction of inspired oxygen (minimum $21 \%$,
}

Dr. Mauricio Orozco Levi, PhD: Servicio de Neumología, Hospital Internacional de Colombia, Fundación Cardiovascular de Colombia, Hospital Internacional de Colombia; Dr. Joaquín Gea i Guiral, PhD: Facultad de Ciencias de la Salud y la Vida, Universidad Pompeu Fabra, Servicio de Neumología. Hospital del Mar IMIM. Barcelona España; Dra. Alba Ramírez Sarmiento, PT, PhD: Unidad de Investigaciones, Servicio de Neumología, Fundación Cardiovascular de Colombia; Dra. Ángela Espitia, TR: Unidad de Terapia Respiratoria, Hospital Internacional de Colombia, Fundación Cardiovascular de Colombia; Dra. María Fernanda Saavedra: Servicio de Neumología, Hospital Internacional de Colombia, Fundación Cardiovascular de Colombia; Dra. Melissa Mogollón: Servicio de Neumología, Hospital Internacional de Colombia, Fundación Cardiovascular de Colombia; Dra. Diana Isabel Cáceres, RN, MsC, PhD: Servicio de Neumología, Hospital Internacional de Colombia, Fundación Cardiovascular de Colombia. Universidad Cooperativa de Colombia; Dr. Rubén Duarte Reyes: Unidad de Cuidado Crítico y Vigilancia Intensiva, Fundación Cardiovascular de Colombia; Dra. Norma Serrano, MsC: Unidad de Investigaciones, Desarrollo e Innovación, Hospital Internacional de Colombia; Ing. Leonardo Rodríguez; Ingeniero Industrial, Hospital Internacional de Colombia, Fundación Cardiovascular de Colombia; Ing. Eugenio Sarmiento: Diseñador Industrial, Unidad de Diseño y Desarrollo, Fundación Cardiovascular de Colombia; Dr. Camilo Pizarro: Unidad de Cuidado Crítico y Vigilancia Intensiva, Fundación Cardiovascular de Colombia. Floridablanca (Colombia).

Correspondencia: Dr. Mauricio Orozco-Levi, Floridablanca (Colombia).

E-mail: mauricioorozcolevi@gmail.com mauricioorozco@fcv.org

Recibido: 16/I/2017 Aceptado: 4 /IV/2018 
maximum 99\%), was conceptualized, designed and constructed. The limits (maximum and minimum) of recommended flow to generate the aerosol are $6-81 / \mathrm{min}$. In no case of use complications occurred.

Conclusions: CONTROLizer is a dual and non-invasive integrated device for the micronebulization of respiratory solutions and continuous control of the fraction of inspired oxygen. Due to its qualities and functioning, the device was perceived as adequate and safe to be applied in healthy individuals and patients admitted in both intensive care and conventional hospitalization rooms.

(Acta Med Colomb 2018; 43: 90-99).

Keywords: oxygen therapy, respiratory failure, hypercapnia, medical devices, micronebulization.

\section{Introducción}

Las enfermedades respiratorias pueden alterar la capacidad de los pulmones para intercambiar tanto el oxígeno como el gas carbónico con el medio. Por esta razón existen métodos artificiales tanto para la administración de oxígeno suplementario como para facilitar la ventilación pulmonar (1). Una de las técnicas más comunes de tratamiento respiratorio es la nebulización de medicamentos para administrarlos por vía inhalada mediante un gas propelente $(2,3)$. El oxígeno presurizado es el gas propelente más utilizado en los dispositivos de micronebulización.

El oxígeno es un fármaco que requiere una dosis para obtener los efectos beneficiosos esperados, pero también puede precipitar efectos adversos difíciles de evitar si no se controla dicha dosis (4). Un problema clínico frecuente es que los pacientes requieran oxígeno suplementario al mismo tiempo que reciben la nebulización de otro fármaco. Esto obliga a nebulizar con oxígeno como propelente, o administrarlo simultáneamente mediante cánulas nasales. Estas estrategias pueden inducir hiperoxia iatrógena y precipitar hipoventilación alveolar, aumento del contenido arterial de $\mathrm{CO}_{2}$ y depresión del sistema nervioso central $(5,6)$. Existe evidencia clínica que $15 \%$ de los pacientes que acuden con insuficiencia respiratoria a un servicio de atención médica desarrollan encefalopatía hipercápnico-hiperóxica grave. Consecuentemente estos pacientes pueden requerir ventilación mecánica, asociándose a incrementos de los costos de atención y de la morbimortalidad (7).

El control de la fracción inspirada de oxígeno puede disminuir el riesgo de hiperoxia durante la nebulización de fármacos. Este control puede obtenerse mediante el uso de dispositivos de tipo Venturi para oxigenoterapia. A los efectos de esta investigación, el principio Venturi produce aspiración de aire ambiente a través de un(os) orificios, dado que el oxígeno en movimiento dentro de un conducto cerrado disminuye su presión y aumenta su velocidad al pasar por una menor sección. El tamaño y disposición de los orificios permite modificar las proporciones de la mezcla. Como resultado se obtendrá una mezcla con menor fracción de oxígeno. Sin embargo, no existe un sistema, hasta donde podemos conocer, que permita nebulizar soluciones y fármacos en respiración espontánea utilizando el oxígeno como propelente y que simultáneamente incluya un control preciso de la fracción inspirada de este gas. Con la intención de resolver este problema clínico, pertinente y relevante, desarrollamos el presente estudio de conceptualización, diseño, prototipado funcional y evaluación perceptual de un dispositivo integrado de micronebulización y control preciso de la fracción inspirada de oxígeno para el uso en humanos, al cual denominamos CONTROLizer.

\section{Diseño del dispositivo dual}

\section{Métodos}

Se diseñó un dispositivo que fue denominado con el acrónimo CONTROLizer (Controlled Oxygen Fraction Nebulizer), que en forma esquemática se muestra en la Figura 1. Inicialmente se realizó un diseño conceptual de un primer componente con base en las propiedades del efecto Venturi que permitiría controlar la fracción inspirada de oxígeno. Posteriormente se diseñó un segundo componente conceptual representado por un recipiente que permitiría obtener una micronebulización de soluciones. Estos dos componentes se analizaron individualmente y ensamblados mediante simulaciones tridimensionales de micronebulización mediante gas presurizado. Esta metodología permitió evaluar múltiples variantes morfológicas y su impacto tanto sobre el flujo de oxígeno como sobre la mezcla. Los cambios de flujo dentro del dispositivo (asumiendo igualdad de flujo de masa a la entrada y a la salida) dependieron principalmente del área de sección transversa.

Componente para mezcla de gases. En la Figura 2 se observa la geometría de los ocho dispositivos Venturi diseñados que mostraban el mejor comportamiento según los resultados del análisis de la mecánica de fluidos. La simulación mediante elementos finitos se inició con una operación de 'mallado' de cada pieza, lo que creó puntos estratégicos (nodos) de análisis, intercomunicados, los cuales formaban una red sobre la cual se evaluó el comportamiento del fluido. Este análisis se realizó con base en la geometría propia de cada dispositivo, sus parámetros de entrada, y las características físicas del fluido y del entorno $(8,9)$. Para tales efectos se utilizó el software especializado SolidWorks (SolidWorks, Dassaut Systèmes, SolidWorks Corporation, Delaware, Massachusets, USA). Se construyeron sendos prototipos funcionales mediante una impresión 3D utilizando como material el ácido poliláctico (PLA). La fase de estudio funcional incluyó la medición de la $\mathrm{FiO}_{2}$ y sus cambios en cada dispositivo tras tres minutos de estabilidad 


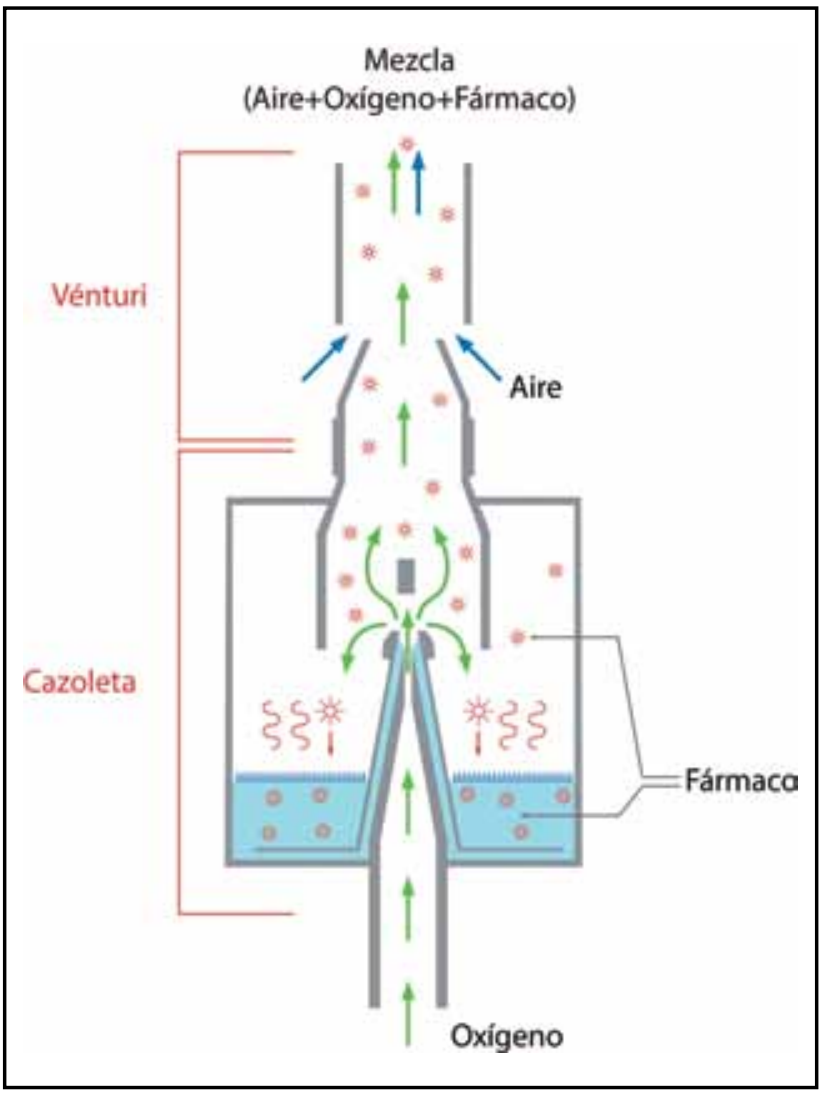

Figura 1. Fundamentos del diseño conceptual de los dos componentes del dispositivo CONTROLizer. Esquema conceptual del nuevo sistema con aplicación dual, dada por el acople en serie de un dispositivo fundamentado en el principio Venturi con un dispositivo para micronebulización basado en aceleración de gas presurizado. El sistema así concebido fue denominado con el acrónimo CONTROLizer. en cada condición mediante un equipo con sensor galvánico de oxígeno (sensibilidad 0-100\%, precisión >2\%) (AII $2000^{a}$-Oxygen Analyzer, Analytical Industries Inc., Pomona, CA, USA). El diseño seleccionado final de acuerdo con los criterios de eficiencia de control de mezcla de oxígeno-aire aparece en la Figura $2 f$.

Componente para micronebulización de fluido. En cuanto al nebulizador, este consta de un recipiente donde se introduce el fármaco por nebulizar, y una tobera interna que genera la nube de gas debido a la aceleración del oxígeno (o aire) que se precipita al pasar por una menor área, localizada en el orificio de la parte superior. La aceleración del gas disminuye su presión al salir de la reducción del orificio, obteniéndose el efecto de succión del líquido contenido en el recipiente. Este líquido sube por capilaridad entre el pequeño espacio que existe entre la superficie de la tobera interna de la boquilla central del recipiente y la superficie interior del cono central de la pieza llamada tobera. El oxígeno es inyectado a una alta presión por el conducto de entrada que posee el recipiente del micronebulizador en su parte inferior. El recipiente del nebulizador incluye demarcación equivalente de 1 , 2, 3, 4, 5 y 6 cc del líquido de acuerdo con el volumen del recipiente. Una vez definido el modelo conceptual se procedió a elaborar un modelo funcional del dispositivo para evaluarlo tanto en individuos sanos como pacientes voluntarios. Finalmente, con los resultados obtenidos en esta fase se elaboraron los moldes para inyección plástica y se continuó con la fase de producción.

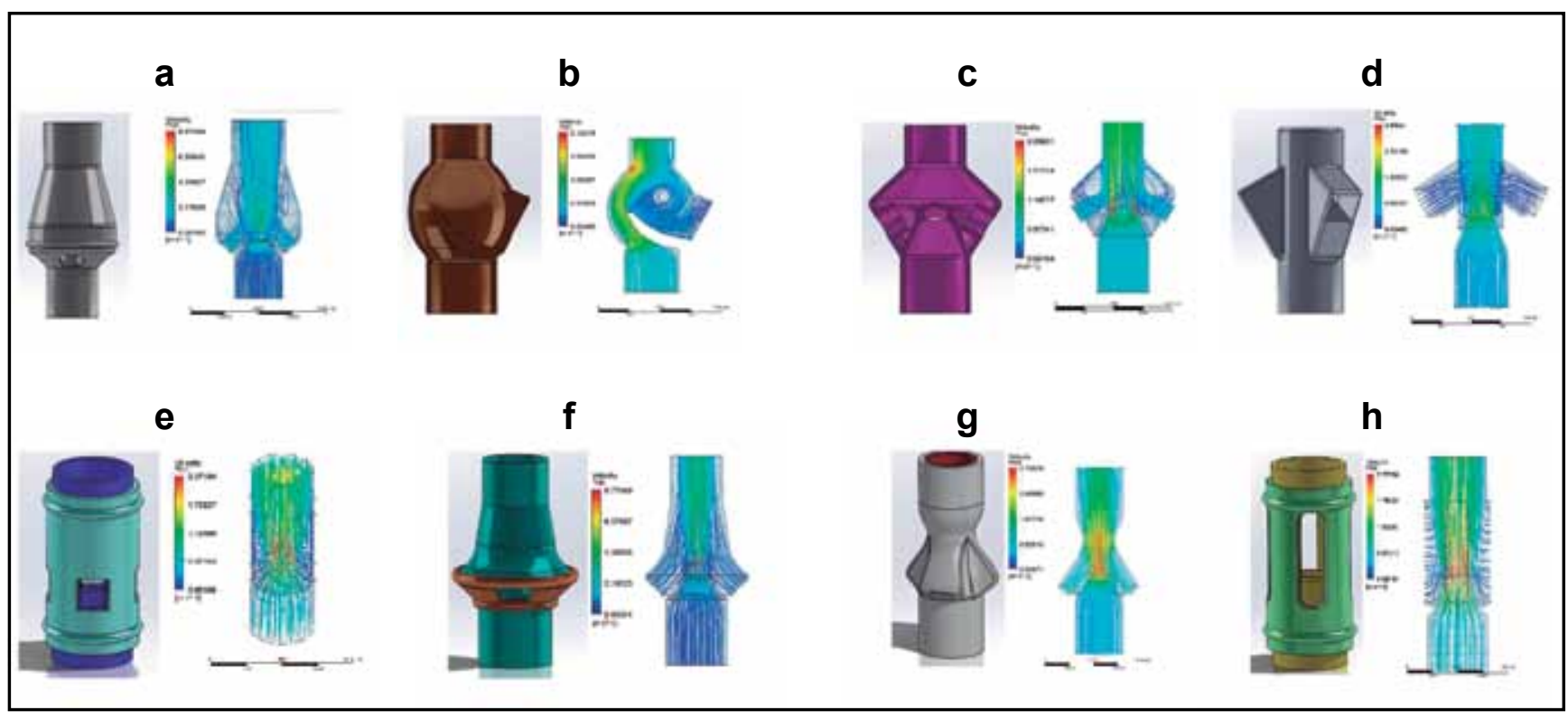

Figura 2. Diseño de los dispositivos de tipo venturi y gráficas demostrativas de flujo interno. Figuras ( $a, b, c, d, e, f, g$ y $h$ ) representativas de los ocho mejores dispositivos de tipo Venturi que se obtuvieron entre una multitud de diseños conceptuales en software. Estos Venturi mostraban mayor eficiencia para obtener mezclas con fluido externo, menor recirculación, y menor turbulencia interna de acuerdo al diseño y estudio dinámico de fluidos bajo software especializado de análisis de elementos finitos (SolidWorks, Dassaut Systèmes, SolidWorks Corporation, Delaware, Massachusets, USA). A continuación se procedió al prototipado mediante impresiones en $3 D$ (material ácido poliláctico) para realizar los estudios funcionales. Al culminar estas fases de estudio, el dispositivo (f) fue el que cumplía con todos los requisitos funcionales predefinidos para la eventual utilización en el escenario clínico. Para más detalles técnicos, favor dirigirse al texto y registro de patente del dispositivo. 
Tabla 1. Relaciones entre el flujo de oxígeno y presión ejercida en el dispositivo CONTROLizer.

\begin{tabular}{|c|c|c|c|c|c|}
\hline \multicolumn{6}{|c|}{ (A) flujo de oxígeno registrado en el caudalímetro y presión $\left(\mathrm{cmH}_{2} \mathrm{O}\right)$ en el dispositivo vacío } \\
\hline Flujo de $\mathrm{O}_{2}(\mathrm{~L} / \mathrm{min})$ & $\mathrm{P1}\left(\mathrm{cmH}_{2} \mathrm{O}\right)$ & $\mathbf{P 2}\left(\mathrm{cmH}_{2} \mathrm{O}\right)$ & $\mathbf{P 3}\left(\mathrm{cmH}_{2} \mathrm{O}\right)$ & Pmedia $\left(\mathrm{cmH}_{2} \mathrm{O}\right)$ & SD \\
\hline 4 & 144.1 & 146.2 & 144.1 & 144.8 & 0.7 \\
\hline 6 & 350.1 & 357.2 & 349.4 & 352.2 & 4.2 \\
\hline 8 & 615.9 & 610.3 & 609.6 & 611.7 & 2.8 \\
\hline 10 & 929.5 & 919.6 & 932.3 & 927.4 & 6.3 \\
\hline 12 & 1040.6 & 1047.6 & 1054.6 & 1047.6 & 7.0 \\
\hline \multicolumn{6}{|c|}{ (B) Flujo de oxígeno registrado en el caudalímetro y presión $\left(\mathrm{cmH}_{2} \mathrm{O}\right)$ en el dispositivo con volumen bajo de solución (2 ce de SF) } \\
\hline Flujo de $O_{2}(\mathrm{~L} / \mathrm{min})$ & $\mathrm{P1}\left(\mathrm{cmH}_{2} \mathrm{O}\right)$ & $\mathrm{P} 2\left(\mathrm{cmH}_{2} \mathrm{O}\right)$ & $\mathrm{P3}\left(\mathrm{cmH}_{2} \mathrm{O}\right)$ & Pmedia $\left(\mathrm{cmH}_{2} \mathrm{O}\right)$ & SD \\
\hline 4 & 151.2 & 149.1 & 151.9 & 150.5 & 1.4 \\
\hline 6 & 376.2 & 369.1 & 377.6 & 374.0 & 4.2 \\
\hline 8 & 652.5 & 636.3 & 643.3 & 644.0 & 7.7 \\
\hline 10 & 822.6 & 815.6 & 808.6 & 815.6 & 7.0 \\
\hline 12 & 914.0 & 986.4 & 892.9 & 930.9 & 48.5 \\
\hline \multicolumn{6}{|c|}{ (C) Flujo de oxígeno registrado en el caudalímetro y presión $\left(\mathrm{cmH}_{2} \mathrm{O}\right)$ en el dispositivo con volumen alto de solución (6 cc de SF) } \\
\hline Flujo de $\mathrm{O}_{2}(\mathrm{~L} / \mathrm{min})$ & $\mathbf{P 1}\left(\mathrm{cmH}_{2} \mathrm{O}\right)$ & $\mathbf{P 2}\left(\mathrm{cmH}_{2} \mathrm{O}\right)$ & $\mathbf{P 3}\left(\mathrm{cmH}_{2} \mathrm{O}\right)$ & Pmedia $\left(\mathrm{cmH}_{2} \mathrm{O}\right)$ & SD \\
\hline 4 & 147.6 & 147.6 & 156.8 & 150.7 & 5.3 \\
\hline 6 & 361.4 & 364.9 & 385.3 & 370.5 & 12.9 \\
\hline 8 & 609.6 & 635.6 & 649.7 & 631.6 & 20.3 \\
\hline 10 & 907.0 & 922.5 & 966.7 & 932.1 & 31.0 \\
\hline 12 & 1089.8 & 1100.3 & 1065.2 & 1085.1 & 18.0 \\
\hline \multicolumn{6}{|c|}{ (D) Flujo de oxígeno registrado en el caudalímetro y presión $\left(\mathrm{cmH}_{2} \mathrm{O}\right)$ ejercida en el dispositivo en las tres condiciones } \\
\hline Flujo de $\mathrm{O}_{2}(\mathrm{~L} / \mathrm{min})$ & $\mathbf{P 1}\left(\mathrm{cmH}_{2} \mathrm{O}\right)$ & $\mathbf{P 2}\left(\mathrm{cmH}_{2} \mathrm{O}\right)$ & P3 $\left(\mathrm{cmH}_{2} \mathrm{O}\right)$ & Pmedia $\left(\mathrm{cmH}_{2} \mathrm{O}\right)$ & SD \\
\hline 4 & 147.6 & 147.6 & 150.9 & 148.7 & 2.5 \\
\hline 6 & 362.6 & 363.7 & 370.8 & 365.6 & 7.1 \\
\hline 8 & 626.0 & 627.4 & 634.2 & 629.1 & 10.3 \\
\hline 10 & 886.4 & 885.9 & 902.5 & 891.7 & 14.8 \\
\hline 12 & 1014.8 & 1044.8 & 1004.2 & 1021.2 & 24.5 \\
\hline \multicolumn{6}{|c|}{$\begin{array}{l}\text { Valores de presión en el dispositivo registradas en su cámara central que se relacionan con el establecimiento de flujo constante de } \mathrm{O}_{2} \text { titulado por caudalímetros. Las mediciones se realizaron } \\
\text { en flujos predeterminados regulares de } 2-12 \mathrm{~L} / \mathrm{min} \text {. Las condiciones incluyeron tanto el dispositivo seco (vacío), como el cargado con volúmenes bajos ( } 2 \text { cc) o altos ( } 6 \text { cc) de solución salina. } \\
\text { Abreviaturas: P, presión. }\end{array}$} \\
\hline
\end{tabular}

\section{Evaluación de los aspectos perceptuales y tolerancia ante el uso del dispositivo}

Población de Estudio. Para la evaluación aspectos perceptuales y seguridad de uso del dispositivo, se definieron como población de estudio a tres grupos de voluntarios, cada uno de diez individuos. El primer grupo estuvo representado por voluntarios sanos, el segundo grupo por pacientes hospitalizados en una unidad convencional de mediana complejidad (patología médica o quirúrgica), y el tercer grupo estuvo representado por pacientes hospitalizados en unidades de cuidados intensivos (patología médica o quirúrgica torácica). Todos fueron incluidos después de 24 horas o más de la extubación en caso que hubiesen requerido ventilación mecánica. Se verificó en todos los casos que se encontraran en condición de estabilidad clínica, capacidad de realizar movimientos respiratorios y corporales generales, y facultades mentales preservadas. En todos los casos se solicitó consentimiento escrito para participar en el estudio.

Criterios de exclusión. Negativa para participar en el estudio, ventilación mecánica, intubación orotraqueal, traqueostomía, presencia de sedación (Ramsay > 2), o medicación miorrelajante, alteración del sensorio o del ánimo, situación clínica de exacerbación no compensada de enfermedades cardiorrespiratorias, dolor no controlado, sangrado reciente o activo, infección respiratoria o de otro órgano activa o no controlada, sepsis, inestabilidad hemodinámica, respiratoria, anímica o neurológica; receptores de trasplante de cualquier órgano, y todas entidades o 
situaciones médicas particulares que pudieran interferir en la capacidad para ofrecer respuestas ante la encuesta.

Protocolo de estudio. Tras obtener el consentimiento informado se procedió a la evaluación y registro de constantes elementales basales (pulsioximetría, frecuencia cardiaca, frecuencia respiratoria y presión arterial sistémica). Los voluntarios realizaron el estudio respirando con el dispositivo en posición sedente o semirrecumbente. En todos los casos se realizó la micronebulización de $5 \mathrm{cc}$ de suero fisiológico estéril mediante el nuevo dispositivo CONTROLizer ante un flujo de $\mathrm{O}_{2}$ de $8 \mathrm{l} / \mathrm{min}$ como propelente hasta completar la nebulización. En los pacientes que recibían oxigenoterapia, el estudio se realizó ante la misma $\mathrm{FiO}_{2}$. Durante dicho proceso se monitorizaron las variables fisiológicas cada minuto. Se contabilizó el tiempo total de la nebulización y se mantuvo la monitorización durante los tres minutos siguientes a terminar el procedimiento. Una vez finalizada la micronebulización, se realizó una encuesta a cada paciente la cual incluía aspectos sobre la percepción del dispositivo en siete dominios: adaptación a la interfase nasobucal, peso, tamaño, molestias en los ojos, disnea, hermeticidad y percepción del tiempo de nebulización, utilizando escalas psicométricas de tipo Osgood (Addendum 1) y Likert (Addendum 2) modificadas. Se registró el diferencial semántico perceptual respecto de función, seguridad y comodidad en un rango que incluyó en ambos casos los extremos "totalmente de acuerdo" hasta "total desacuerdo" (10).

Análisis estadístico. Los resultados se presentan como valor de la media y dispersión (desviación estándar). La estimación de la significación estadística se realizó mediante comparación de datos pareados o independientes, según el caso, para variables no-paramétricas. El análisis estadístico de los resultados fue realizado por los epidemiólogos vinculados al área de diseño y desarrollo de nuestra institución.

\section{Resultados}

Prototipo funcional, validación y diseño definitivo del dispositivo CONTROLizer. El aspecto general del sistema y sus componentes se muestran en la Figura 3. El diseño del componente de micronebulización se basó en un recipiente, el cual implica alta presión continua del gas propelente para establecer flujo hasta de 10/min. El flujo de entrada ideal para la micronebulización de suero fisiológico se encuentra en el rango de 6-8/min (Figura 4). Las relaciones entre el flujo de gas y las presiones generadas dentro del dispositivo de micronebulización aparecen especificadas en la Tabla 1. El componente de regulación continua de la fracción de oxígeno se acopló en el extremo distal del recipiente . Se regula la $\mathrm{FiO}_{2}$ de salida mediante un sistema de cierre de las ventanas laterales al Venturi entre 21-99\%. Existen múltiples posiciones intermedias del elemento regulador que, al estar demarcados, permiten modificar y controlar la $\mathrm{FiO}_{2}$ que se entrega al paciente. $\mathrm{La} \mathrm{FiO}_{2}$ se mantiene estable ante cambios de posición extremos entre

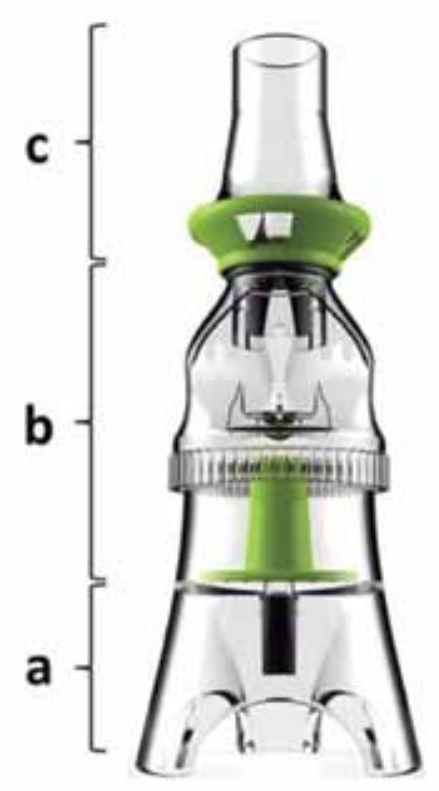

Figura 3. Aspecto general del dispositivo y sus componentes funcionales. Aspecto general del sistema de micronebulización con control de la fracción inspirada de $\mathrm{O}_{2}$. Se muestran los tres elementos funcionales del dispositivo definidos como (a) conector a la tubuladura procedente de la fuente de gas a presión; (b) recipiente de micronebulización de las soluciones líquidas mediante aceleración de alta velocidad del gas propelente; $y(c)$ elemento de control de la mezcla del gas propelente (aire comprimido u $\mathrm{O}_{2}$ ) con aire ambiental $\left(\mathrm{FIO}_{2}=0,21\right)$ para modificar la fracción de $\mathrm{O}_{2}$ que el dispositivo puede aportar al paciente. Para más especificaciones técnicas remitirse al apartado de Métodos.

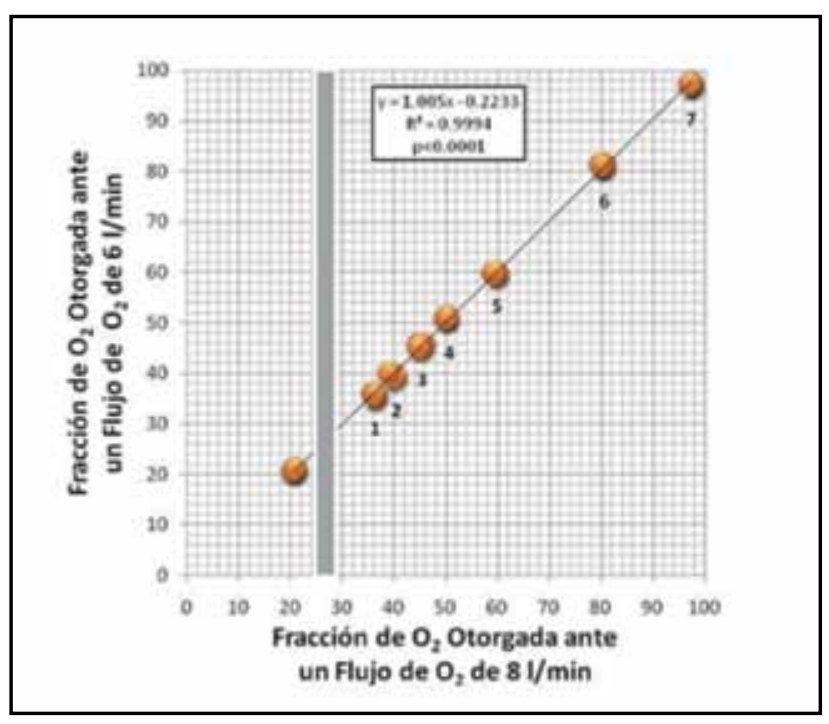

Figura 4. Fracción de $O$ otorgada por el dispositivo ante flujos constantes de 6 y $8 \mathrm{l} / \mathrm{min}$ de O absoluto. Gráfico de dispersión que muestra la correlación de los valores de fracción de $\mathrm{O}_{2}$ otorgada por el dispositivo cuando se utiliza como propelente el $\mathrm{O}_{2}$ absoluto de una fuente hospitalaria convencional regulada mediante caudalímetros de pared. Mientras menor sea el grado de apertura de las toberas, menor es la entrada de aire ambiental $(\mathrm{FiO}=0,21)$ y viceversa, basado en el principio fundamental del efecto Venturi. Las fracciones inspiradas de $\mathrm{O}_{2}$ pueden ser modificadas desde un dintel mínimo de $21 \%$ hasta un máximo cercano al $100 \%$. Los valores se ubican en su totalidad muy cercanos a la línea de identidad entre 6 y $8 \mathrm{l} / \mathrm{min}$. Estos resultados implican recomendar el uso del dispositivo en este rango de flujo entrante de $\mathrm{O}_{2}$ absoluto. Si el propelente es aire comprimido, la fracción de $\mathrm{O}_{2}$ será idéntica a la ambiental $(0,21)$. Abreviaturas: Ref, fracción de $\mathrm{O}_{2}$ que se registra al utilizar aire comprimido; 1-7, codificación numérica arbitraria de las posiciones secuenciales de cierre de las toberas del dispositivo que permiten aumentar progresivamente la fracción entregada de $\mathrm{O}_{2}$. 
verticalidad $\left(90^{\circ}\right)$ e inversión a $180^{\circ}\left(\right.$ cambio $\left.\mathrm{FiO}_{2}<0,5 \%\right)$, así como ante el uso continuado durante horas. Para una descripción más detallada del dispositivo, el lector puede remitirse al documento de patente disponible en internet con acceso público (11).

Aspectos perceptuales y tolerancia del uso del dispositivo. La nebulización de suero fisiológico fue bien tolerada en todos los pacientes y no se presentaron cambios de relevancia clínica en las constantes vitales elementales de frecuencia cardiaca, presión arterial sistémica, frecuencia respiratoria, y saturación de la oxihemoglobina capilar. No se observaron cambios de relevancia clínica en ninguna de estas variables. Metodológicamente se exigió por consenso que el prototipo final se consideraría válido cuando obtuviera calificaciones perceptuales mayores al dintel mínimo predefinido en todos los dominios y todos los voluntarios (sanos y enfermos). En la escala de Osgood la media del valor perceptual de los siete dominios analizados fue $4.5 \pm 1.1$ en voluntarios sanos y $5.1 \pm 0.9$ en los enfermos. Resulta de interés que se detectaron diferencias en varios de los dominios evaluados a expensas de valores superiores en el grupo pacientes. En la escala de Likert, los valores medios de los dominios fueron de $3.1 \pm 0.9$ en los voluntarios sanos, y $3.8 \pm 0.2$ en los pacientes hospitalizados. También en este caso la estimación de la diferencia perceptual alcanzó significación estadística en diferentes dominios, con valores consistentemente mayores en el grupo pacientes. Para aportar un acercamiento en términos de comparabilidad de las dos escalas, se realizó una normalización de los valores en términos de porcentaje relativo al valor máximo posible en cada escala (Figura 5). En la escala Osgood, el promedio de las siete variables analizadas fue similar en ambas evaluaciones $(5.1 \pm 0.9$ en la primera y $5.5 \pm 0.3$ en la segunda). En la misma línea, la escala Likert mostró un promedio acumulado para las siete variables de $3.8 \pm 0.2$ u.a. en la primera medición, y $3.7 \pm 0.3$ en la segunda medición (n.s). Estos resultados permiten considerar que las respuestas han sido concordantes intra-individualmente entre las dos mediciones. Un punto importante por resaltar es que se habían realizado previamente dos estudios perceptuales (datos no incluidos) con prototipos previos que denotaron calificación por debajo del dintel aceptable de 2 en el dominio de 'fugas o goteo'. Aunque los demás dominios fueron calificados siempre con valores $>2$ estos datos implicaron un rediseño y mejoras de construcción en el sistema de cierre del recipiente de los prototipos previos, lo que permitió resolver dichas fugas. En consecuencia, la última evaluación por duplicado del funcionamiento del prototipo obtuvo ya calificaciones medias muy por encima del dintel mínimo considerado previamente como aceptable.

\section{Discusión}

El presente estudio describe el proceso de invención, desarrollo y validación perceptual de un sistema dual de micronebulización con control preciso de la $\mathrm{FIO}_{2}$, denominado

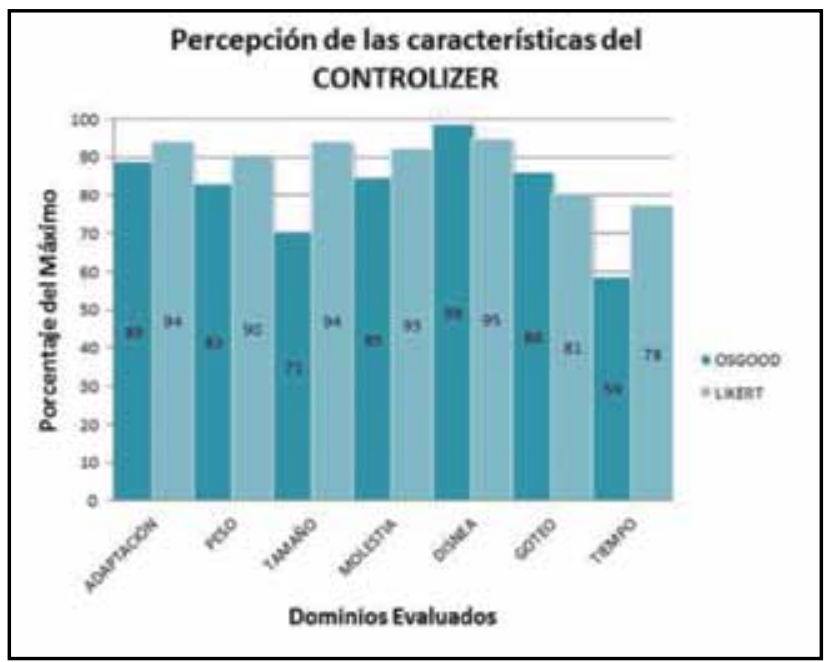

Figura 5. Percepción de las características del dispositivo por parte de los individuos sanos y pacientes. Resultados normalizados (en términos de porcentaje respecto del máximo posible en la escala) de la percepción de las siete características del CONTROLIZER por parte de los usuarios (tanto sanos como pacientes). La figura incluye los resultados de las dos escalas utilizadas (Osgood y Likert). Es de resaltar que el 100\% de las características evaluadas fueron calificadas por encima del valor mínimo aceptable, predefinido por el grupo investigador.

CONTROLizer. Este dispositivo representa una solución razonable para la administración de tratamientos por vía inhalada y que permite mezclar de forma regulada aire con oxígeno durante el tiempo en que se nebulizan fármacos en solución. El sistema ha sido validado técnicamente en el laboratorio de bioingeniería demostrando una alta precisión y reproducibilidad en su funcionamiento. Además se ha comprobado la bondad del dispositivo en cuanto a aspectos perceptuales y seguridad, tanto en sujetos sanos como en pacientes ingresados en diversos niveles de atención.

Desde un punto de vista clínico, el resultado más relevante del presente estudio es que el uso del nuevo dispositivo otorga la posibilidad de controlar de forma precisa y permanente la fracción inspirada de oxígeno que se administra a los pacientes durante la micronebulización de fármacos (12). Es posible que con esta forma de terapia se pueda prevenir el desarrollo de las complicaciones graves derivadas de la hipercapnia e hiperoxia en pacientes con enfermedades respiratorias. Esto es especialmente relevante, ya que no es posible identificar con precisión a los pacientes más susceptibles de desarrollar eventos adversos ante la administración de concentraciones elevadas de $\mathrm{O}_{2}$. Algunos estudios previos de nuestro grupo (datos no publicados) han identificado que la hiperoxia iatrógena parece ser un aspecto fundamental en la evolución desfavorable de pacientes con EPOC grave descompensada, durante las primeras horas de atención. El oxígeno tiene efectos deletéreos a dosis elevadas sobre ciertos pacientes respiratorios $(13,14) \mathrm{y}$, sin embargo, este efecto es a menudo infravalorado en algunos ámbitos de atención (p.e. servicios de urgencias). Un aspecto complementario de asegurar una fracción inspiratoria de oxígeno preestablecida y constan- 
te, es su potencial impacto en el sistema de cobertura de salud, reduciendo el riesgo de que los pacientes requieran escalonamiento de las terapias respiratorias, así como prolongación de su estancia hospitalaria (15). Un segundo punto de interés clínico tiene que ver con la versatilidad del nuevo dispositivo en tres usos terapéuticos independientes o simultáneos. En primer lugar, el dispositivo permite administrar oxigenoterapia de forma más segura; segundo, permite la humidificación de secreciones en pacientes en que se haya indicado dicha medida; y tercero, permite la micronebulización de fármacos. Esta versatilidad deriva de que el dispositivo puede usarse para la oxigenoterapia de forma continuada sin incluir necesariamente la nebulización. Además, el sistema de humidificación convencional con vaso (adosado convencionalmente al caudalímetro de pared) también puede ser utilizado con el nuevo dispositivo. Una ventaja adicional del nuevo dispositivo es que puede utilizarse también conectándolo a compresores de aire ambiente para realizar la terapia respiratoria con humidificación y medicamentos nebulizados sin utilizar oxígeno como propelente. Por todo lo anterior, el nuevo dispositivo podría simplificar el manejo de los distintos elementos sin necesidad de intercambiar conexiones. Esto representará un consecuente ahorro en tubuladuras y disminución de los riesgos de la manipulación de las mismas.

Un tercer punto de interés clínico tiene relación con la posibilidad de aplicación del dispositivo en escenarios de diversos niveles de complejidad. Su sencillez permite el uso tanto en áreas de actividad asistencial especializada, como en recursos de asistencia ambulatoria y atención básica de salud en zonas urbanas o rurales. Elementos más prosaicos pero también importantes son la facilidad de manejo del dispositivo, sus elementos resistentes a la humedad, a los golpes y la corrosión, su solidez y reducido peso, la presencia de bordes romos en todos sus elementos, y su bajo coste. Por otra parte, los materiales plásticos utilizados cumplen con todas las normativas de bioseguridad para ser utilizados en seres humanos y pueden ser de un solo uso.

Cabe destacar que la evaluación de los aspectos perceptuales fue realizada tanto en voluntarios sanos como en pacientes hospitalizados. Además, estos últimos se hallaban ingresados en diversos niveles de complejidad de la atención (cuidados intensivos y sala de hospitalización convencional), correspondientes además a aquellos lugares en que suelen emplearse los dispositivos de oxigenoterapia y nebulización. Esto permitió disponer de las opiniones de sus potenciales usuarios en las diversas fases del diseño, lo que se complementó con la monitorización de las variables fisiológicas, permitiendo determinar el impacto y eventuales efectos adversos en términos objetivos. En cuanto a las dos escalas psicométricas hay que resaltar su validez en la evaluación perceptual de las características físicas y funcionales del dispositivo. Se trata de unas escalas robustas, utilizadas ampliamente en estudios de investigación psicométrica cualitativa ante un elemento definido. Sus mediciones en sistemas septuagesimal o sexagesimal permiten obtener elementos de evaluación tanto de percepciones como de sensaciones. Finalmente, otro punto por resaltar es la evidencia de que todos los voluntarios valoraron el prototipo final por encima del valor mínimo establecido previamente como válido. Esto permite asumir que no se requerían ya más modificaciones en sus características físicas (forma, tamaño, color, peso) o de funcionamiento (molestias, fugas). En conjunto se dispone así de una información funcional y perceptual robusta, que permite establecer que el diseño final del CONTROLizer es seguro, adecuado, suficiente, cómodo y práctico.

El presente estudio tiene algunas limitaciones que deben ser mencionadas. Aunque no disponemos aún de estudios farmacoeconómicos todo parece sugerir que el potencial impacto de la utilización del dispositivo no incrementará los costes asistenciales de la terapia respiratoria. Al contrario, puede disminuirlos al evitar complicaciones. Algunas de las limitaciones del presente estudio están representadas por la ausencia de información en cuanto al rendimiento del sistema de micronebulización en términos de distribución espacial de las partículas, diámetro de masa mediana aerodinámica, ni otros parámetros en cuanto a calidad del aerosol. Al igual que en los demás recipientes de micronebulización existentes, el componente de nebulización del dispositivo no incluye un sistema activo de recirculación o filtro para partículas de mayor tamaño. Finalmente, futuros estudios de coste-eficiencia tendrán un interés central para la eventual difusión del sistema, que ya ha sido patentado a nivel del país de origen.

\section{Conclusiones}

CONTROLizer es un dispositivo funcional y eficaz para el control de la fracción de $\mathrm{O}_{2}$ inspirada durante la nebulización de soluciones de fármacos. Así mismo, el dispositivo es percibido en términos de cualidad y funcionamiento como adecuado y seguro por individuos sanos y pacientes ingresados tanto en salas de cuidados intensivos como de hospitalización convencional. Las complicaciones y accidentes durante su ensamblaje, carga y nebulización han sido nulos. En consecuencia, se trata de un dispositivo que puede ofrecer un sistema de oxigenoterapia segura y nebulización apropiada a pacientes con enfermedades respiratorias, tanto en régimen ambulatorio como institucionalizados.

\section{Consideraciones éticas}

El estudio de análisis perceptual del dispositivo en pacientes e individuos sanos fue aprobado por todos los investigadores, así como el Comité de Ética y ensayos clínicos de la institución. Todos los voluntarios firmaron un consentimiento informado después de recibir información respecto de los objetivos del estudio y sus aspectos procedimentales.

\section{Financiación}

Subvencionado, en parte, por CONVENIO SENA-FCV Ref. 00453-2012. Plan de Fortalecimiento Institucional COLCIENCIAS, Ref. 477-2012, y COLCIENCIAS 734-2013, Proyecto Exención de Impuestos COLCIENCIAS, Ref. Contrato 656624037813- 2013. 


\section{Conflicto de intereses}

Los autores declaran que poseen la patente del dispositivo CONTROLizer hasta el año 2033 de acuerdo con el certificado de patentes $\mathrm{N}^{\circ} 13-256191-00003-0000$ de la superintendencia de Industria y Comercio de la República de Colombia.

\section{Agradecimientos}

A todos los voluntarios sanos y pacientes que aceptaron desinteresadamente participar en los estudios; Dr. Víctor Castillo, MD, Presidente de la Fundación Cardiovascular de Colombia-Hospital Internacional de Colombia, y Sonia Ramírez Ribero, Vicepresidente Corporativo, por su liderazgo, tenacidad y activa participación en la consolidación del proyecto; Gloria Parra, RN, MSc, por su valiosa participación en la logística de los estudios en pacientes; Terapeutas Respiratorias Olga Nova y Durley Carvajal por su participación en algunos de los estudios en voluntarios; Auxiliares de Enfermería (SENA) Pamela Peña y Yurley Hernández por su participación en algunas de las evaluaciones perceptuales del dispositivo.

\section{Referencias}

1. West JB. Fisiología respiratoria, 7a Edición. ISBN 9788479034931. Editorial Panamericana, 2005.

2. Bott J, Blumenthal S, Buxton M, Ellum S, Falconer C, Garrod R. British Thoracic Society Physiotherapy Guideline Development Group. Guidelines for the physiotherapy management of the adult, medical, spontaneously breathing patient. Thorax 2009; 64 Supl 1:i1-51.

3. Gardenhire DS, Ari A, Hess DR, Myers TR. A guide to aerosol delivery devices for respiratory therapists 3rd Ed, American Association for Respiratory Care, 2013.

4. Markovitz GH, Colthurst J, Storer TW, Cooper CB. Effective inspired oxygen concentration measured via transtracheal and oral gas analysis. Respir Care 2010; 55: 453-459.
5. Gea J, Orozco-Levi M, Gallart LI. Increased inspiratory oxygen fractions ( $\mathrm{FiO} 2)$ using a conventional delivery nebulizer. Arch Bronconeumol. 2010;46: 230-237.

6. Kallet RH, Matthay MA. Hyperoxic Acute Lung Injury. Respir Care 2013; 58:123-141.

7. Higgins S. Oxygen versus medical air for nebulisers in patients with COPD. Evidence Centre Critical Appraisal. Series 2003: Therapy. Clayton (Australia): Monash University; p. 1-8.

8. Wong W, Fletcher DF, Traini D, Chan H-K, Young PM. The use of computational approaches in inhaler development. Advanced Drug Delivery Reviews 2012; 64: $312-322$.

9. Perdigones F, Luque A, Quero JM. PDMS microdevice for precise liquid aspiration in the submicroliter range based on the Venturi effect. Microelectronic Engineering 2010; 87: 2103-2109.

10. Streiner D, Norman G, Cairney J. Health Measurement Scales: A Practical Guide to Their Development and Use. 5th ed.,2015, Oxford University Press, Great Clarendon. ISBN: 9780199685219.

11. http://serviciospub.sic.gov.co/Sic/ServiciosPI/consultaDocs/index.php?lsxiozaqwscersderwerrteyr=pol\%F1 mkjuiutdrsesdfrcdfds\&lspm=1NfdyNXK079 vYWRZ1+PWyMjht5aZbmWepJqcmpW5oZ6lktvPzcymj3ZYk6Ch3M3bxM3 Yk2JW1KLN18rG3eSTZmFiWdnX3dXc1LuobWFZz9PMy8rSyqdtV3bY3NzY1eO3pG10otfh3s/d0Mg

12. Rau JL, Ari A, Restrepo RD. Performance comparison of nebulizer designs: constant-output, breath-enhanced, and dosimetric. Respir Care 2004; 49: 174-179.

13. Ward JJ. High-Flow Oxygen Administration by Nasal Cannula for Adult and Perinatal Patients. Respir Care 2013; 58: 98-122.

14. Pierson DJ. Oxygen in Respiratory Care: A Personal Perspective from 40 Years in the Field. Respir Care 2013; 58: 196-204.

15. Dolovich MB, Ahrens RC, Hess DR. Device selection and outcomes of aerosol therapy: evidence-based guidelines: American College of Chest Physicians/ American College of Asthma, Allergy, and Immunology. Chest 2005; 127:335- 371. 


\section{Addenda}

\section{Addendum 1. Escala OSGOOD de siete dominios.}

\section{Enunciados de diferencial semántico:}

Pregunta 1: La adaptación del sistema a su cara es...

Pregunta 2: El peso del equipo es....

Pregunta 3: El tamaño del equipo es...

Pregunta 4: Las molestias en los ojos durante la nebulización son...

Pregunta 5: La dificultad respiratoria durante la nebulización es...

Pregunta 6: La fuga y goteo del equipo es...

Pregunta 7: El tiempo de nebulización es...

\section{Intervalo de diferencial semántico: \\ Valor $=0: \quad$ Muy difícil - pesado - grande. \\ Valor $=1: \quad$ Bastante difícil - pesado - grande. \\ Valor $=2 . \quad$ Ligeramente difícil - pesado - grande . \\ Valor $=3: \quad$ Ni lo uno ni lo otro. \\ Valor $=4: \quad$ Ligeramente fácil - liviano - pequeño. \\ Valor $=5: \quad$ Bastante fácil - liviano - pequeño. \\ Valor $=6: \quad$ Muy fácil - liviano - pequeño.}

Matriz utilizada para la evaluaciòn sumaria:

\begin{tabular}{|c|c|c|c|c|c|c|c|}
\hline \multicolumn{8}{|c|}{ Escala psicométrica 1} \\
\hline Escala OSGOOD & $\begin{array}{c}\text { Muy } \\
6\end{array}$ & $\begin{array}{c}\text { Bastante } \\
\quad 5\end{array}$ & $\begin{array}{l}\text { Leve } \\
4\end{array}$ & $\begin{array}{c}\text { Ni lo uno } \\
\text { ni lo otro } \\
3\end{array}$ & $\begin{array}{l}\text { Leve } \\
2\end{array}$ & $\begin{array}{c}\text { Bastante } \\
1\end{array}$ & $\begin{array}{c}\text { Muy } \\
0\end{array}$ \\
\hline $\begin{array}{l}\text { La adaptación de la } \\
\text { máscara a su cara es... }\end{array}$ & Muy fácil & $\begin{array}{l}\text { Bastante } \\
\text { fácil }\end{array}$ & $\begin{array}{l}\text { Ligeramente } \\
\text { fácil }\end{array}$ & $\begin{array}{l}\text { Ni lo uno ni } \\
\text { lo otro }\end{array}$ & $\begin{array}{l}\text { Ligeramente } \\
\text { dificil }\end{array}$ & $\begin{array}{l}\text { Bastante } \\
\text { difícil }\end{array}$ & Muy dificil \\
\hline El peso del equipo es... & Muy liviano & $\begin{array}{c}\text { Bastante } \\
\text { liviano }\end{array}$ & $\begin{array}{l}\text { Ligeramente } \\
\text { liviano }\end{array}$ & $\begin{array}{l}\text { Ni lo uno ni } \\
\text { lo otro }\end{array}$ & $\begin{array}{l}\text { Ligeramente } \\
\text { pesado }\end{array}$ & $\begin{array}{l}\text { Bastante } \\
\text { pesado }\end{array}$ & Muy pesado \\
\hline $\begin{array}{l}\text { El tamaño del equipo } \\
\text { es... }\end{array}$ & $\begin{array}{c}\text { Muy } \\
\text { pequeño }\end{array}$ & $\begin{array}{l}\text { Bastante } \\
\text { pequeño }\end{array}$ & $\begin{array}{l}\text { Ligeramente } \\
\text { pequeño }\end{array}$ & $\begin{array}{l}\text { Ni lo uno ni } \\
\text { lo otro }\end{array}$ & $\begin{array}{l}\text { Ligeramente } \\
\text { grande }\end{array}$ & $\begin{array}{l}\text { Bastante } \\
\text { grande }\end{array}$ & Muy grande \\
\hline $\begin{array}{l}\text { Las molestias en los ojos } \\
\text { son... }\end{array}$ & Ninguna & Escasas & Pocas & $\begin{array}{l}\text { Ni lo uno ni } \\
\text { lo otro }\end{array}$ & Algunas & Bastantes & Muchas \\
\hline $\begin{array}{l}\text { La dificultad respiratoria } \\
\text { es... }\end{array}$ & Ninguna & Escasa & Poca & $\begin{array}{l}\text { Ni lo uno ni } \\
\text { lo otro }\end{array}$ & Leve & Bastante & Muy intensa \\
\hline $\begin{array}{l}\text { La fuga y goteo del } \\
\text { líquido es... }\end{array}$ & Ninguna & Escasa & Poca & $\begin{array}{l}\text { Ni lo uno ni } \\
\text { lo otro }\end{array}$ & Leve & Bastante & Muy intensa \\
\hline $\begin{array}{l}\text { El tiempo de la } \\
\text { nebulización... }\end{array}$ & Muy corto & $\begin{array}{l}\text { Bastante } \\
\text { corto }\end{array}$ & $\begin{array}{l}\text { Ligeramente } \\
\text { corto }\end{array}$ & $\begin{array}{l}\text { Ni lo uno ni } \\
\text { lo otro }\end{array}$ & $\begin{array}{l}\text { Ligeramente } \\
\quad \text { largo }\end{array}$ & $\begin{array}{l}\text { Bastante } \\
\text { largo }\end{array}$ & Muy largo \\
\hline
\end{tabular}




\section{Addendum 2. Escala likert de siete dominios.}

\section{Enunciados de diferencial semántico.}

Pregunta 1: La máscara se adapta fácilmente a su cara.

Pregunta 2: El peso del equipo es cómodo.

Pregunta 3: El tamaño del equipo es cómodo.

Pregunta 4: La nebulización no produce molestias en los ojos.

Pregunta 5: La nebulización no produce dificultad respiratoria.

Pregunta 6: El equipo sufre fugas y goteos.

Pregunta 7: El tiempo empleado en la nebulización fue adecuado.

\section{Escala de valoración especifica.}

Valor $=0: \quad$ Totalmente en desacuerdo.

Valor $=1: \quad$ En desacuerdo

Valor $=2$ : $\quad$ Ni de acuerdo ni en desacuerdo

Valor $=3$ : $\quad$ De acuerdo

Valor $=4: \quad$ Totalmente de acuerdo

\section{Matriz para la evaluaciòn sumaria:}

\begin{tabular}{|c|c|c|c|c|c|}
\hline \multicolumn{6}{|c|}{ Escala psicométrica 2} \\
\hline Escala LIKERT & $\begin{array}{c}\text { Totalmente en } \\
\text { desacuerdo } \\
0\end{array}$ & $\begin{array}{c}\text { En desacuerdo } \\
1\end{array}$ & $\begin{array}{l}\text { Ni de acuerdo ni } \\
\text { en desacuerdo } \\
2\end{array}$ & $\begin{array}{l}\text { De acuerdo } \\
3\end{array}$ & $\begin{array}{c}\text { Totalmente de } \\
\text { acuerdo } \\
4\end{array}$ \\
\hline La máscara se adapta fácilmente a la cara & $\begin{array}{l}\text { Totalmente en } \\
\text { desacuerdo }\end{array}$ & En desacuerdo & $\begin{array}{l}\text { Ni de acuerdo ni } \\
\text { en desacuerdo }\end{array}$ & De acuerdo & $\begin{array}{l}\text { Totalmente de } \\
\text { acuerdo }\end{array}$ \\
\hline El peso del equipo es cómodo & $\begin{array}{l}\text { Totalmente en } \\
\text { desacuerdo }\end{array}$ & En desacuerdo & $\begin{array}{l}\text { Ni de acuerdo ni } \\
\text { en desacuerdo }\end{array}$ & De acuerdo & $\begin{array}{l}\text { Totalmente de } \\
\text { acuerdo }\end{array}$ \\
\hline El tamaño del equipo es cómodo & $\begin{array}{l}\text { Totalmente en } \\
\text { desacuerdo }\end{array}$ & En desacuerdo & $\begin{array}{l}\text { Ni de acuerdo ni } \\
\text { en desacuerdo }\end{array}$ & De acuerdo & $\begin{array}{l}\text { Totalmente de } \\
\text { acuerdo }\end{array}$ \\
\hline $\begin{array}{l}\text { La nebulización no produce molestias en } \\
\text { los ojos }\end{array}$ & $\begin{array}{l}\text { Totalmente en } \\
\text { desacuerdo }\end{array}$ & En desacuerdo & $\begin{array}{l}\text { Ni de acuerdo ni } \\
\text { en desacuerdo }\end{array}$ & De acuerdo & $\begin{array}{l}\text { Totalmente de } \\
\text { acuerdo }\end{array}$ \\
\hline $\begin{array}{l}\text { La nebulización no produce dificultad } \\
\text { respiratoria }\end{array}$ & $\begin{array}{l}\text { Totalmente en } \\
\text { desacuerdo }\end{array}$ & En desacuerdo & $\begin{array}{l}\text { Ni de acuerdo ni } \\
\text { en desacuerdo }\end{array}$ & De acuerdo & $\begin{array}{l}\text { Totalmente de } \\
\text { acuerdo }\end{array}$ \\
\hline El equipo sufre fugas y goteos & $\begin{array}{l}\text { Totalmente en } \\
\text { desacuerdo }\end{array}$ & En desacuerdo & $\begin{array}{l}\text { Ni de acuerdo ni } \\
\text { en desacuerdo }\end{array}$ & De acuerdo & $\begin{array}{l}\text { Totalmente de } \\
\text { acuerdo }\end{array}$ \\
\hline El tiempo de nebulización es adecuado & $\begin{array}{l}\text { Totalmente en } \\
\text { desacuerdo }\end{array}$ & En desacuerdo & $\begin{array}{l}\text { Ni de acuerdo ni } \\
\text { en desacuerdo }\end{array}$ & De acuerdo & $\begin{array}{l}\text { Totalmente de } \\
\text { acuerdo }\end{array}$ \\
\hline
\end{tabular}

